\title{
Expanded Very Large Array observations of the H66 $\alpha$ and He66 $\alpha$ recombination lines toward MWC 349A
}

\author{
Laurent Loinard and Luis F. Rodríguez \\ Centro de Radioastronomía y Astrofísica, Universidad Nacional Autónoma de México \\ Apartado Postal 3-72, 58090, Morelia, Michoacán, México (l.loinard@crya.unam.mx)
}

\begin{abstract}
We have used the greatly enhanced spectral capabilities of the Expanded Very Large Array to observe both the $22.3 \mathrm{GHz}$ continuum emission and the H66 $\alpha$ recombination line toward the well-studied Galactic emission-line star MWC 349A. The continuum flux density is found to be $411 \pm 41 \mathrm{mJy}$ in good agreement with previous determinations. The H66 $\alpha$ line peak intensity is about $25 \mathrm{mJy}$, and the average line-to-continuum flux ratio is about $5 \%$, as expected for local thermodynamic equilibrium conditions. This shows that the H66 $\alpha$ recombination line is not strongly masing as had previously been suggested, although a moderate maser contribution could be present. The He66 $\alpha$ recombination line is also detected in our observations; the relative strengths of the two recombination lines yield an ionized helium to ionized hydrogen abundance ratio $y^{+}=0.12 \pm 0.02$. The ionized helium appears to share the kinematics of the thermally excited ionized hydrogen gas, so the two species are likely to be well mixed. The electron temperature of the ionized gas in MWC 349A deduced from our observations is $6,300 \pm 600 \mathrm{~K}$.
\end{abstract}

Subject headings: circumstellar matter — radio lines: stars — stars: emission line, $\mathrm{Be}$ — stars: individual (MWC 349A) — stars: mass loss

\section{Introduction}

MWC 349A is a peculiar B[e] star which happens to be the brightest continuum source of its kind at $\mathrm{cm}$ wavelengths (e.g. Braes et al. 1972; Tafoya et al. 2004). It is located in the direction of the Cyg OB2 association, at a distance which has been estimated between $1.2 \mathrm{kpc}$ (Cohen et al. 1985) and $1.7 \mathrm{kpc}$ (Hofmann et al. 2002). MWC349A may have a B0 III companion (named MWC 349B), located 2!"4 to its west (Merrill et al. 1932; Cohen et al. 1985). The cm radio continuum emission from MWC 349A can be successfully described in terms of an isothermal wind photoevaporating from the surface of a neutral Keplerian disk surrounding a young massive star (Lugo et al. 2004). 
MWC 349A also exhibits hydrogen recombination line emission detectable from the radio (Altenhoff et al. 1981) to the optical (Hartmann et al. 1980). The intensity of the recombination lines at $\mathrm{cm}$ wavelengths was found to be consistent with nearly thermal, LTE conditions (e.g. Altenhoff et al. 1981; Escalante et al. 1989; Rodríguez \& Bastian 1994). However, in a remarkable discovery, Martín-Pintado et al. (1989a) found that the mm recombination lines towards MWC 349A were emitting in a maser mode, and were therefore tens of times stronger than expected for thermal conditions. This discovery was rapidly confirmed, and it is now known that all the hydrogen recombination lines at $\mathrm{mm}$, sub-mm, far-IR and mid-IR wavelengths (from $\mathrm{H} 39 \alpha$ at about 100 $\mathrm{GHz}$ to $\mathrm{H} 7 \alpha$ at about 16,000 GHz) are masing (Gordon et al. 2001; Thum et al. 1994a; Strelnitski et al. 1996a, 1996b; Thum et al. 1998). These maser lines are also known to be time variable (Martín-Pintado et al. 1989b; Gordon et al. 2001). To this date, MWC 349A remains the only source known to show such high-gain natural hydrogen maser emission. The line amplification has been investigated theoretically by Thum et al. (1994b) and Ponomarev et al. (1994), and is believed to arise in the inner region of a nearly edge-on disk where the eastern region is redshifted with respect to the western one (Planesas et al. 1992; Weintroub et al. 2008).

In an unexpected turn of events, Martín-Pintado et al. (1993) reported that the H66 $\alpha$ recombination line at $22 \mathrm{GHz}$ was about 5 times stronger than expected from thermal emission, and argued that this line must, therefore, also be masing. This was surprising and implied that the maser effect could still be present at wavelengths as large as $1.3 \mathrm{~cm}$. The observations reported by MartínPintado et al. (1993), however, were taken with the Very Large Array (VLA) at the time when it only had limited spectral capabilities. In particular, the observations were made with a total velocity coverage of only about $160 \mathrm{~km} \mathrm{~s}^{-1}$, barely enough to include the entire width of the H66 line. This restriction could have led to an improper continuum subtraction and to an overestimate of the line intensity. In this Letter, we take advantage of the greatly enhanced spectral capabilities of the Expanded Very Large Array (EVLA) to reobserve this important source and obtain accurate H66 $\alpha$ line parameters.

\section{Observations}

The data were collected with the Expanded Very Large Array in its most compact (D) configuration on June 16, 2010 while 22 antennas were available in the array. Five of these antennas had to be flagged because they consistently delivered low visibility amplitudes or because they exhibited significant phase jumps. As a consequence, the final data products were constructed from observations collected on 17 antennas only. The correlator was set up to deliver 256 spectral channels, each $500 \mathrm{kHz}$ wide, centered on the rest frequency of the H66 $\alpha$ line at $22.364 \mathrm{GHz}$. This corresponds to a total velocity coverage in excess of $1,700 \mathrm{~km} \mathrm{~s}^{-1}$ (about 10 times the velocity cov- 
erage of Martín-Pintado et al. 1993), and a spectral resolution of about $6.7 \mathrm{~km} \mathrm{~s}^{-1}$. The data were calibrated with the Common Astronomy Software Applications (CASA) package following standard procedures. The bandpass was measured using observations of J1743-0350 and J0319+4130 (3C84) obtained at the beginning and the end of the observations. The flux scale was set using an observation of J0137+3309 (3C48). Finally, the amplitude and phase gains were monitored using observations of $\mathrm{J} 2015+3710$ (with a bootstrapped flux density of $2.3 \mathrm{Jy}$ ) obtained roughly every 10 minutes throughout the 3 hours of observations.

Both the continuum and the $\mathrm{H} 66 \alpha$ line were clearly detected in the observations, so two data sets were created from the calibrated visibilities. A continuum data set was generated by averaging 137 line-free spectral channels (corresponding to an equivalent bandwidth of about $70 \mathrm{MHz}$ ). Simultaneously, a continuum-free spectral line data set was created by subtracting a constant continuum (measured over the same 137 line-free channels) from the initial data. Both data sets were then imaged using robust weighting and 0.5 arcsecond pixels. The resulting angular resolution was $3^{\prime \prime} .35 \times 2$ '. 07 ; P.A. $-60.7^{\circ}$. To improve the overall calibration, the continuum data set was selfcalibrated -in phase only- and the resulting gain corrections were applied both to the continuum and the continuum-free spectral line data sets which were then re-imaged.

\section{Results}

The continuum image shows the presence of a strong point-like source at the expected position of MWC 349A $\left(\alpha_{J 2000.0}=20^{\mathrm{h}} 32^{\mathrm{m}} 45.52 ; \delta_{J 2000.0}=40^{\circ} 39^{\prime} 36^{\prime \prime}\right.$ '62 $)$. A 2D Gaussian fit to this source yields an integrated flux of $411 \mathrm{mJy}$ with a formal error of a fraction of a mJy. This error, however, does not account for the systematic uncertainty on the absolute flux calibration. We estimate this systematic uncertainty to be about $10 \%$, and conclude that the $22.3 \mathrm{GHz}$ flux of MWC 349A is $F_{v}=411 \pm 41 \mathrm{mJy}$. This value is in good agreement with the flux obtained in previous $1.3 \mathrm{~cm}$ observations of MWC 349A. For instance, Tafoya et al. (2004) reported a $22.4 \mathrm{GHz}$ flux density of $446.2 \pm 44.8 \mathrm{mJy}$. We note, also, that changes in the morphology of MWC $349 \mathrm{~A}$ at $\mathrm{cm}$ wavelengths are known to have occurred (Rodríguez et al. 2007), so moderate variations in its flux density might also be present.

In the continuum-free spectral data set, emission is clearly detected in more than 40 contiguous spectral channels around the frequency of the H66 $\alpha$ line. This emission remains unresolved at the angular resolution of our observations, so a single-pixel spectrum at the peak position of the source (Figure 1) provides a complete description of the line emission. This spectrum shows a very prominent double-peaked feature surrounded by a $1,200 \mathrm{~km} \mathrm{~s}^{-1}$ emission-free spectral baseline. This baseline is remarkably linear but not flat. Instead, it shows a slope which most certainly results from the combined effect of the true positive spectral index of MWC 349A (e.g. Tafoya et 
al. 2004) and the uncorrected negative spectral indices of the flux and bandpass calibrators 1 A spectrum with a linear baseline removed is shown in Figure 1b. The VLA observations reported by Martín-Pintado et al. (1993) only covered the spectral bandwidth shown by the thick horizontal bracket on Figure 1b. It is clear that this spectral window barely contains the most prominent spectral line detected here, and does not include at all the weaker feature seen at more negative velocities. Since observations with such a limited velocity coverage would contain no channels free of line emission, it would clearly be nearly impossible to properly subtract a continuum from them.

A simultaneous fit to the spectrum with two Gaussian components (blue solid line in Figure 1b) provides the parameters listed in Table 1 . The separation in velocity between the two spectral features is $130.4 \pm 4.4 \mathrm{~km} \mathrm{~s}^{-1}$, very similar to the separation $\left(122.2 \mathrm{~km} \mathrm{~s}^{-1}\right)$ expected between the hydrogen and helium $66 \alpha$ recombination lines. Indeed, a fit where the separation between the two spectral components is constrained to be $122.2 \mathrm{~km} \mathrm{~s}^{-1}$ (red solid line in Figure 1b) is very nearly as good as a fit where the separation can vary freely (the parameters corresponding to that constrained fit are also given in Table 1). We conclude that the weaker spectral feature seen at more negative velocities in our spectrum is the He66 $\alpha$ line. We note that Thum et al. (1992) also identified a blue-shifted feature in their $\mathrm{H} 41 \alpha$ spectrum of MWC 349A, and favored an interpretation where that additional component is the $\mathrm{He} 41 \alpha$ line. The present results clearly lend strong support to that interpretation. Interestingly, however, the He41 $\alpha$ line detected by Thum et al. (1992) appears to be at a somewhat more negative radial velocity than its hydrogen counterpart $\left(V_{l s r}=-31 \mathrm{~km} \mathrm{~s}^{-1}\right.$ vs. $\left.V_{l s r}=+1 \mathrm{~km} \mathrm{~s}^{-1}\right)$. If our unconstrained fit is taken at face value, the He66 $\alpha$ line is also at a more negative radial velocity than the $\mathrm{H} 66 \alpha$ line $\left(V_{l s r}=-15.2 \pm 4.3 \mathrm{~km} \mathrm{~s}^{-1} \mathrm{vs} . V_{l s r}=-7.0 \pm 0.7\right.$ $\mathrm{km} \mathrm{s}^{-1}$ ), but the difference is significantly less than for the $41 \alpha$ lines. We will come back to that point in Section 4.3. We note, finally, that the H66 $\alpha$ line also shows a hint of excess emission on the redshifted side that may be related to the winds predicted in the models of Avalos \& Lizano (in preparation).

\section{Discussion}

\subsection{Excitation of the $\mathrm{H66} \alpha$ line}

The H66 $\alpha$ line to continuum ratio reported by Martín-Pintado et al. (1993) is typically about $20 \%$. In contrast, the line peak temperature found here $(25 \mathrm{mJy})$ is only about $6 \%$ of the underlying

\footnotetext{
${ }^{1}$ In the future, CASA will be able to take into account the spectral dependences of the calibrators, and it will become possible to measure directly the spectral index of the target from observations such as those presented here.
} 

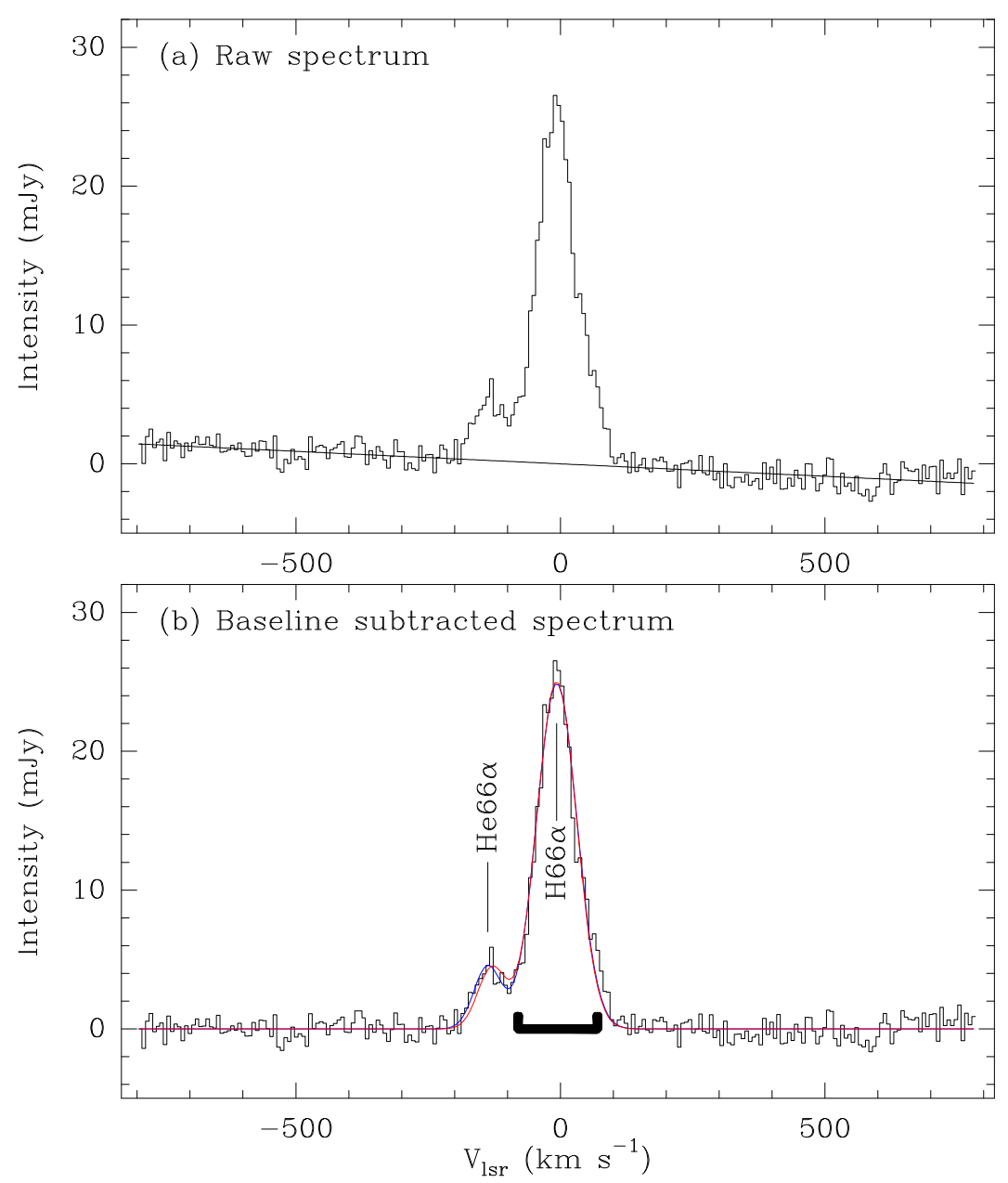

Fig. 1.- (a) Raw spectrum of MWC 349A centered on the rest frequency of the H66 $\alpha$ line. The best fit to the slope in the spectral baseline with a straight line is shown as a solid black line. (b) Baseline-subtracted spectrum. The velocity coverage of the VLA data presented by Martín-Pintado et al. (1993) is shown as a horizontal bracket below the spectral lines. The blue solid line shows a fit with two unconstrained Gaussian components. The red solid line shows a similar fit but with the velocity separation between the two components constrained to be $122.2 \mathrm{~km} \mathrm{~s}^{-1}$, the expected velocity separation between the $\mathrm{H} 66 \alpha$ and the $\mathrm{He} 66 \alpha$ lines.

continuum. A somewhat more robust line to continuum ratio of about $5 \%$ is obtained by averaging over the central $90 \mathrm{~km} \mathrm{~s}^{-1}$ of the line. In LTE conditions and adopting a full width at half power of $90 \mathrm{~km} \mathrm{~s}^{-1}$ and an electron temperature of $8,000 \mathrm{~K}$, the H66 $\alpha$ line is predicted to represent about $5 \%$ of the underlying continuum (Altenhoff et al. 1981; Rodríguez 1982; Martín-Pintado 2002) in excellent agreement with the observed values. We conclude that the H66 $\alpha$ line in MWC 349A is 
Table 1: Spectral line parameters

\begin{tabular}{|c|c|c|c|}
\hline & $S_{V}(\mathrm{mJy})$ & $V_{l s r}\left(\mathrm{~km} \mathrm{~s}^{-1}\right)$ & $\Delta V\left(\mathrm{~km} \mathrm{~s}^{-1}\right)^{\mathrm{a}}$ \\
\hline \multicolumn{4}{|l|}{ Unconstrained fit: } \\
\hline Feature 1 & $24.9 \pm 0.3$ & $-7.0 \pm 0.7$ & $90.0 \pm 1.7$ \\
\hline Feature 2 & $4.5 \pm 0.5$ & $-137.4 \pm 4.3$ & $61.3 \pm 9.9$ \\
\hline \multicolumn{4}{|l|}{ Constrained fit: } \\
\hline $\mathrm{H} 66 \alpha$ & $25.0 \pm 0.3$ & $-7.1 \pm 0.7$ & $88.7 \pm 1.6$ \\
\hline He66 $\alpha$ & $4.4 \pm 0.4$ & 11 & $62.8 \pm 8.2$ \\
\hline
\end{tabular}

${ }^{a} \Delta V$ is the full width at half maximum.

largely thermally excited and is not strongly masing as had been suggested by Martín-Pintado et al. (1993).

One could argue, however, that our results and those of Martín-Pintado et al. (1993) are not necessarily incompatible because the two sets of observations might trace different regions of MWC 349A. In particular, the present observations were obtained in the most compact (D) configuration of the EVLA and are, therefore, sensitive to even fairly extended emission. If it existed, such extended emission would have been filtered out in the observations reported by Martín-Pintado et al. (1993), because they were collected in the most extended (A) configuration of the VLA. Could our observations be dominated by extended, thermally excited gas, and those of Martín-Pintado et al. (1993) by denser, more compact masing regions?

This appears not to be the case for the following reasons. At a wavelength of $1.3 \mathrm{~cm}$, the (E)VLA in the A configuration is sensitive to any structure more compact than about 2.5 arcseconds 2 . Our observations, on the other hand, show that the line emission detected here originates in a region more compact than about 1.5 arcseconds. Thus, there are no spatial scales contributing to the emission reported here and to which the observations by Martín-Pintado et al. (1993) were not sensitive. The two sets of observations trace essentially the same gas. Moreover, at $1.3 \mathrm{~cm}$, the size of MWC 349A is about $0.2 \times 00^{\prime \prime} 2$ (e.g. Tafoya et al. 2004; Rodríguez et al. 2007; Martín-Pintado et al. 1993). Since the angular resolution of the (E)VLA at $1.3 \mathrm{~cm}$ in the A configuration is about 0 "'1, MWC 349A only contains 4 independent resolution elements at that wavelength. Thus, the four spectra reported by Martín-Pintado et al. (1993) in their Figure 2 trace most of the extent of

\footnotetext{
${ }^{2}$ http://evlaguides.nrao.edu/index.php?title=Category:Status
} 
the source, and the continuum emission integrated over these four positions will be about $300 \mathrm{mJy}$ (the total continuum emission estimated by Martín-Pintado et al. 1993). According to the spectra shown in Figure 2 of Martín-Pintado et al. (1993), the mean intensity of the H66 $\alpha$ line over the velocity range from -60 to $+50 \mathrm{~km} \mathrm{~s}^{-1}$ is about $20 \%$ of the underlying continuum. The line intensity integrated over these 4 positions must therefore be about $60 \mathrm{mJy}$. As a consequence, the integrated line flux measured over any larger area should be at least $60 \mathrm{mJy}$ in that velocity range 3 Yet, in our spectrum (corresponding to a 3 !' $35 \times 2$ "'07 beam), the flux density is systematically smaller than $25 \mathrm{mJy}$; averaged over the -60 to $+50 \mathrm{~km} \mathrm{~s}^{-1}$ velocity range, it is $18 \mathrm{mJy}$. We conclude that Martín-Pintado et al. (1993) have overestimated the line intensity by a factor 3 to 4.

We can only speculate about the exact reasons which led Martín-Pintado et al. (1993) to overestimate the H66 $\alpha$ line intensity in MWC 349A. In principle, the lack of line-free channels in their observations should have led them to over-estimate the continuum flux (since the channels on the edge of the bandpass used to calculate the continuum still contained line emission) and to underestimate the line to continuum ratio (rather than the other way around). Instead, a more subtle effect, also related to the narrow bandwidth of their observations might have been the culprit. The velocity channels on the edge of the VLA bandpass are well known to be very noisy. A continuum measurement based on these channels may have seriously underestimated the continuum flux density, and adversely affected the line to continuum ratio measurement.

\subsection{Helium abundance and electron temperature}

Our results show that the excitation of the H66 $\alpha$ line very nearly corresponds to LTE conditions. In this situation, the line behaves as if it were approximately optically thin (see Rodríguez 1982). Given its similar excitation and lower abundance, the He66 $\alpha$ line will also behave as if it were approximately optically thin, so the integrated line intensity ratio provides a direct measure of the ionized helium over ionized hydrogen abundance ratio $y^{+}$. From the unconstrained fit (Section 3 ), we obtain $y^{+}=0.12 \pm 0.02$, in good agreement with the value $(0.10 \pm 0.01)$ found by Thum et al. (1992) for the $41 \alpha$ lines.

As a consequence of its relative abundance, the opacity of the helium lines is expected to be about 10 times less than that of the hydrogen lines, so the helium lines are not anticipated to be masing (even at mm wavelengths). This presumably explains why the helium lines associated with strongly masing hydrogen lines at $\mathrm{mm}$, sub-mm, far-IR and mid-IR wavelengths have not been

\footnotetext{
${ }^{3}$ Recall that flux densities measured in Jy do not get diluted: if a 1 Jy source is present at the center of a given field, the total flux density taken over any area containing the source will be $1 \mathrm{Jy}$, independently of how large the chosen area might be.
} 
detected in existing observations. Since the hydrogen lines are tens of times stronger than thermal, the helium to hydrogen line intensity ratio will typically be less than $1 \%$ at mm wavelengths. The noise level of the mm observations was almost certainly adapted to detect the hydrogen lines, and was insufficient to reach the much weaker helium lines.

The high quality of the data presented here allows us to estimate the electron temperature of the ionized gas in MWC 349A. Following Rodríguez et al. (2009), and assuming LTE conditions, the electron temperature, $T_{e}^{*}$, of an ionized, partially optically-thick outflow is given by:

$$
\left[\frac{T_{e}^{*}}{K}\right]=\left[\frac{4700}{\left(1+y^{+}\right)} \frac{S_{C}}{S_{L}}\left(\frac{v_{L}}{G H z}\right)^{1.1}\left(\frac{\Delta v}{k m s^{-1}}\right)^{-1}\right]^{0.87}
$$

where $v_{L}$ is the line frequency, $S_{C}$ is the continuum flux density, $S_{L}$ is the hydrogen peak line flux density, and $\Delta v$ is the hydrogen FWHM line width. Using the helium abundance estimated above and the line and continuum parameters measured in Section 3, we obtain $T_{e}^{*}=6,300 \pm 600 \mathrm{~K}$. This value is consistent with those determined from other radio observations (White \& Becker 1985; Cohen et al. 1985; Escalante et al. 1989).

\subsection{Relative kinematics of the hydrogen and helium lines}

The kinematics of the ionized gas associated with MWC 349A presents several problems that require additional research. For instance, the direction of rotation determined from the $\mathrm{cm}$ thermal lines (eastern side blueshifted and western side redshifted; Rodríguez \& Bastian 1994) is opposite to that found in the mm maser lines (Planesas et al. 1992; Weintroub et al. 2008). Also, while the thermal radio recombination lines at $\mathrm{cm}$ wavelengths peak at negative velocities (typically $V_{l s r} \sim$ $-20 \mathrm{~km} \mathrm{~s}^{-1}$; e.g. Rodríguez \& Bastian 1994), the centroid of maser mm radio recombination lines is at positive velocities $\left(V_{l s r} \sim+10 \mathrm{~km} \mathrm{~s}^{-1}\right.$; e.g. Weintroub et al. 2008).

In our unconstrained fit, the LSR velocity of the He66 $\alpha$ line appears to be $-15.2 \pm 4.3 \mathrm{~km} \mathrm{~s}^{-1}$ whereas the LSR velocity of the He41 $\alpha$ line reported by Thum et al. (1992) is $-31 \pm 8 \mathrm{~km} \mathrm{~s}^{-1}$. Thus, on average, the Helium lines appear to peak at $V_{l s r} \sim-20 \mathrm{~km} \mathrm{~s}^{-1}$, similar to the velocity of the thermal $\mathrm{cm}$ lines of hydrogen. This strongly suggests that the helium lines originate in the same gas as the thermal cm hydrogen lines.

The H66 $\alpha$ line detected here peaks at a velocity $\left(-7.0 \pm 0.7 \mathrm{~km} \mathrm{~s}^{-1}-\right.$ Section 2$)$, intermediate between the $+10 \mathrm{~km} \mathrm{~s}^{-1}$ masing mm lines, and the $-20 \mathrm{~km} \mathrm{~s}^{-1}$ thermal $\mathrm{cm}$ lines. A plausible (albeit somewhat ad hoc) explanation is that the H66 $\alpha$ line is largely dominated by a thermal component at $-20 \mathrm{~km} \mathrm{~s}^{-1}$ but also contains a moderate masing component around $+10 \mathrm{~km} \mathrm{~s}^{-1}$. 
The reversed situation might occur with the $\mathrm{H} 41 \alpha$ line at $92 \mathrm{GHz}$ reported by Thum et al. (1992) which peaks at $+1.0 \pm 0.9 \mathrm{~km} \mathrm{~s}^{-1}$. In that second case, the emission might be dominated by the masing line at $+10 \mathrm{~km} \mathrm{~s}^{-1}$ but with a moderate thermal contribution. It would be interesting to analyze further the relative kinematics of the hydrogen and helium lines, for instance by obtaining high resolution EVLA observations of the $53 \alpha$ and $66 \alpha$ lines at 0.7 and $1.3 \mathrm{~cm}$, respectively.

\section{Conclusions and perspectives}

We have presented new EVLA observations of the $22.3 \mathrm{GHz}$ continuum and the H66 $\alpha$ line emissions toward the well-studied Galactic emission-line star MWC 349A. The continuum flux density (411 $\pm 41 \mathrm{mJy})$ is in good agreement with previous determinations. The intensity of the $\mathrm{H} 66 \alpha$ line (25 mJy) and the line-to-continuum flux density ratio (5\%), on the other hand, are those expected for local thermodynamic equilibrium. This shows that the H66 $\alpha$ line excitation is largely thermal, and that the line is not strongly masing as had been proposed by Martín-Pintado et al. (1993). The intermediate radial velocity of the $H 66 \alpha$ line, however, might suggest the existence of a moderate maser component. The $\mathrm{He} 66 \alpha$ recombination line is also detected in our observations, and shows that the ionized helium to ionized hydrogen abundance ratio in MWC 349A is $y^{+}=$ $0.12 \pm 0.02$. Because of their shared kinematics, it is likely that the ionized helium gas and the thermally excited ionized hydrogen gas are well mixed. The electron temperature of that gas is $6,300 \pm 600 \mathrm{~K}$.

High-resolution EVLA observations of this and other recombination lines (particularly the $\mathrm{H} 53 \alpha$ at $0.7 \mathrm{~cm}$ ) might help resolve some of the pending issues concerning the kinematics of the gas in MWC 349A. On a more general note, we would like to stress that the present observations demonstrate the enormous potential of the EVLA to observe wide spectral lines. When previous VLA observations had to rely on $a d$ hoc and often very uncertain procedures to remove a spectral baseline, the present observations provide a very clean identification of the line and extremely reliable determinations of the line parameters.

We thank Clemens Thum and an anonymous referee for thoughtful comments on our manuscript, and Vivek Dhawan, Lorant Sjouwerman, Rick Perley, George Moellenbrock, and Michael Rupen for their help and advices at various stage of the data calibration. We acknowledge the support of DGAPA, UNAM, and of CONACyT (México). LL is indebted to the Guggenheim Memorial Foundation for financial support. 


\section{REFERENCES}

Altenhoff, W. J., Strittmatter, P. A., \& Wendker, H. J. 1981, A\&A, 93, 48

Braes, L. L. E., Habing, H. J., \& Schoenmaker, A. A. 1972, Nature, 240, 230

Cohen, M., Bieging, J. H., Welch, W. J., \& Dreher, J. W. 1985, ApJ, 292, 249

Escalante, V., Rodríguez, L. F., Moran, J. M., \& Cantó, J. 1989, Revista Mexicana de Astronomia y Astrofisica, 17, 11

Gordon, M. A., Holder, B. P., Jisonna, L. J., Jr., Jorgenson, R. A., \& Strelnitski, V. S. 2001, ApJ, 559,402

Hartmann, L., Jaffe, D., \& Huchra, J. P. 1980, ApJ, 239, 905

Hofmann, K.-H., Balega, Y., Ikhsanov, N. R., Miroshnichenko, A. S., \& Weigelt, G. 2002, A\&A, 395,891

Lugo, J., Lizano, S., \& Garay, G. 2004, ApJ, 614, 807

Martín-Pintado, J., Bachiller, R., Thum, C., \& Walmsley, M. 1989a, A\&A, 215, L13

Martín-Pintado, J., Bachiller, R., \& Thum, C. 1989b, A\&A, 222, L9

Martín-Pintado, J. 2002, Cosmic Masers: From Proto-Stars to Black Holes, Edts: Victor Mineese and Mark Reid, San Francisco: Astronomical Society of the Pacific, 206, 226

Martín-Pintado, J., Gaume, R., Bachiller, R., Johnston, K., \& Planesas, P. 1993, ApJ, 418, L79

Merrill, P. W., Humason, M. L., \& Burwell, C. G. 1932, ApJ, 76, 156

Planesas, P., Martín-Pintado, J., \& Serabyn, E. 1992, ApJ, 386, L23

Ponomarev, V. O., Smith, H. A., \& Strelnitski, V. S. 1994, ApJ, 424, 976

Rodriguez, L. F. 1982, Revista Mexicana de Astronomia y Astrofisica, 5, 179

Rodriguez, L. F., \& Bastian, T. S. 1994, ApJ, 428, 324

Rodríguez, L. F., Gómez, Y., \& Tafoya, D. 2007, ApJ, 663, 1083

Rodríguez, L. F., Zapata, L. A., \& Ho, P. T. P. 2009, ApJ, 692, 162

Strelnitski, V., Haas, M. R., Smith, H. A., Erickson, E. F., Colgan, S. W. J., \& Hollenbach, D. J. 1996a, Science, 272, 1459 
Strelnitski, V. S., Ponomarev, V. O., \& Smith, H. A. 1996b, ApJ, 470, 1118

Tafoya, D., Gómez, Y., \& Rodríguez, L. F. 2004, ApJ, 610, 827

Thum, C., Martin-Pintado, J., \& Bachiller, R. 1992, A\&A, 256, 507

Thum, C., Matthews, H. E., Harris, A. I., Tacconi, L. J., Schuster, K. F., \& Martín-Pintado, J. 1994a, A\&A, 288, L25

Thum, C., Matthews, H. E., Martín-Pintado, J., Serabyn, E., Planesas, P., \& Bachiller, R. 1994b, A\&A, 283, 582

Thum, C., Martín-Pintado, J., Quirrenbach, A., \& Matthews, H. E. 1998, A\&A, 333, L63

Weintroub, J., Moran, J. M., Wilner, D. J., Young, K., Rao, R., \& Shinnaga, H. 2008, ApJ, 677, 1140

White, R. L., \& Becker, R. H. 1985, ApJ, 297, 677 